\title{
STM-induced light emission from vacuum-evaporated gold film
}

\author{
J U AHAMED ${ }^{1, *}$, S KATANO ${ }^{2}$ and Y UEHARA ${ }^{2}$ \\ ${ }^{1}$ Department of Applied Physics, Electronics and Communication Engineering, University of Chittagong, \\ Chittagong 4331, Bangladesh \\ ${ }^{2}$ Research Institute of Electrical Communication, Tohoku University, Sendai 980-8577, Japan
}

MS received 8 January 2015; accepted 13 April 2015

\begin{abstract}
A vacuum evaporation system has been used to evaporate gold film on glass substrate in order to probe the scanning tunneling microscope-light emission (STM-LE) from the evaporated film. The surface morphology of the evaporated Au film has been checked by atomic force microscope (AFM). In order to estimate the appropriate thickness of the Au film, which is essential for the enhancement of STM-LE in the prism-coupled geometry, a theoretical calculation has been performed. Our theoretical simulation revealed that the light emission from the prism-coupled STM junction is strongly enhanced when the Au film has a thickness of $40 \mathrm{~nm}$. AFM observation also showed that the morphology of the gold films strongly depends on the cleanliness of glass substrates and the deposition temperature. Relatively smooth surface was observed when a 40-nm-thick Au film was evaporated at room temperature on the preannealed glass substrate. Finally, the evaporated films were deposited on the flat bottom of a hemispherical glass prism, and STM-LE from the tip-sample gap into the vacuum (tip-side emission) and into the prism (prism-side emission) were measured. It was found from the experimental results that the prism-side emission is much stronger than the tip-side emission by virtue of the enhancement of the prism-coupled geometry.
\end{abstract}

Keywords. Vacuum evaporation; Au film; morphology; AFM; STM-LE; enhancement.

\section{Introduction}

The nucleation, growth and orientation of thin metal films began very interesting shortly after the first experiment on the interaction between electron beams and crystals $^{1}$ and the interpretation of electron diffraction (ED) patterns. ${ }^{2}$ Brück $^{3}$ studied the influence of the substrate on the orientation of deposited metal films. Using polished quartz plates or smooth molybdenite (both polycrystalline) as substrates, oriented polycrystalline $\mathrm{Au}$ films even at high substrate temperatures $\left(500-700^{\circ} \mathrm{C}\right)$ were obtained. When condensed on quartz at $480^{\circ} \mathrm{C}$, the gold showed an orientation with the $\{111\}$ plane parallel to the surface, and without any preferred azimuthally direction. On freshly cleaved, heated single-crystal rocksalt substrates, areas of single-crystal gold were readily obtained. A marked dependence upon the temperature of the substrates was also reported. Rüdiger ${ }^{4}$ studied the metallic films that were obtained by condensation on various heated surfaces, including mica and quartz. The obtained results confirmed the dependence of the orientation on the substrate temperature, as reported by Brück. Gold evaporated onto heated mica at a temperature of $300-400^{\circ} \mathrm{C}$ revealed crystallites of octahedral faces,

*Author for correspondence (ahamed.jamal@cu.ac.bd) azimuthally oriented within the film. It was also reported that the texture was poorer in thin films with respect to that observed in thick films.

The surface structure of thin metallic films plays an important role for various interfacial phenomena, e.g., in electrochemistry and the chemical physics of thin film superstructures. Evidently, the interfacial structure is of interest, both on the microscopic and on the more macroscopic length scales. ${ }^{5}$ For example, Langmuir-Blodgett (LB) monolayers are typically $2 \mathrm{~nm}$ thick and are intended to coat a solid substrate with a monomolecular film over macroscopic dimensions. Therefore, the flatness and perfection of the substrate on these scales are of major importance.

Scanning tunneling microscope light emission (STMLE) spectroscopy ${ }^{6,7}$ and atomic force microscopy (AFM) ${ }^{8,9}$ add a new dimension to the powerful means of surface investigations, which enable one to explore the energy levels of individual surface nanostructures with atomicscale spatial resolutions. ${ }^{6,7}$ Analysing the spectrum of visible light emitted from the tip-sample gap region provides us information about the nature of the electronic transitions that are localized under the STM tip. Particularly, preparations of the atomically flat and electrical conductive Au films are important to examine the enhancement of light emission via the excitation of localized surface plasmons (LSPs) and surface plasmon polaritons (SPPs). 
Although STM-LE spectroscopy is a powerful tool for nanometre scale investigations of individual surface nanostructures, ${ }^{10-12}$ light to be detected is fundamentally weak owing to the low efficiency of excitation by tunneling current in the range of nanoamperes. As a result, researchers frequently experience difficulty in measuring STM-LE. Hence, it is preferable to improve the signal levels of STM-LE. It was previously reported ${ }^{13}$ that the signal level can be improved by measuring STM-LE in a prism-coupled configuration. In this configuration, the sample is the metallic thin film evaporated on the bottom of a hemispherical glass prism. Then STM-LE can be measured on both sides of the sample. That is to say, the STM-LEs from the tip-sample gap into the vacuum (tipside emission) and into the prism (prism-side emission) can also be performed.

In this paper, at first, we reported the results of an AFM study of Au films thermally evaporated onto microscope-covered glass substrates. Theoretical calculation was carried out in order to estimate the most appropriate thickness of the Au layer for the investigation of STMLE. Finally, we demonstrated the STM-LE measurements of the 40-nm-thick evaporated $\mathrm{Au}$ film in the prismcouple geometry.

\section{Experimental}

\subsection{Preparation of $\mathrm{Au}$ film}

Figure 1 shows the schematic illustrations of vacuum evaporation system used for the preparation of the $\mathrm{Au}$ thin films. The system is divided into five basic sections: a diffusion pump system, a rotary pump, a chamber consisting of a bell jar and hoist, system controls and cabinetry. A liquid-nitrogen cold-trap is also present in the evaporation system. In this deposition technique, a resistive evaporation method was used. Resistive evaporation is a commonly used vacuum deposition process in which electrical energy is used to heat a filament which in turn heats a deposition material to the point of evaporation. In this system, a molybdenum boat is heated electrically with a large current to evaporate the gold wire for the preparation of the metallic $(\mathrm{Au})$ films. During deposition, the film thickness was gradually monitored by using a quartz crystal oscillator.

In order to prepare the Au films on glass substrates, gold wire was cleaned with ethanol in an ultrasonic bath. Glass substrates were also cleaned in two ways: first, they were washed by detergent and pure water and then cleaned with ethanol in an ultrasonic bath; finally they were cleaned with acetone in an ultrasonic bath and then dried under a stream of nitrogen. Cleaned gold wire was put into the molybdenum evaporation boat by bending it into a suitable length. Before evaporation, the glass substrates were preannealed at $450^{\circ} \mathrm{C}$ for several hours in

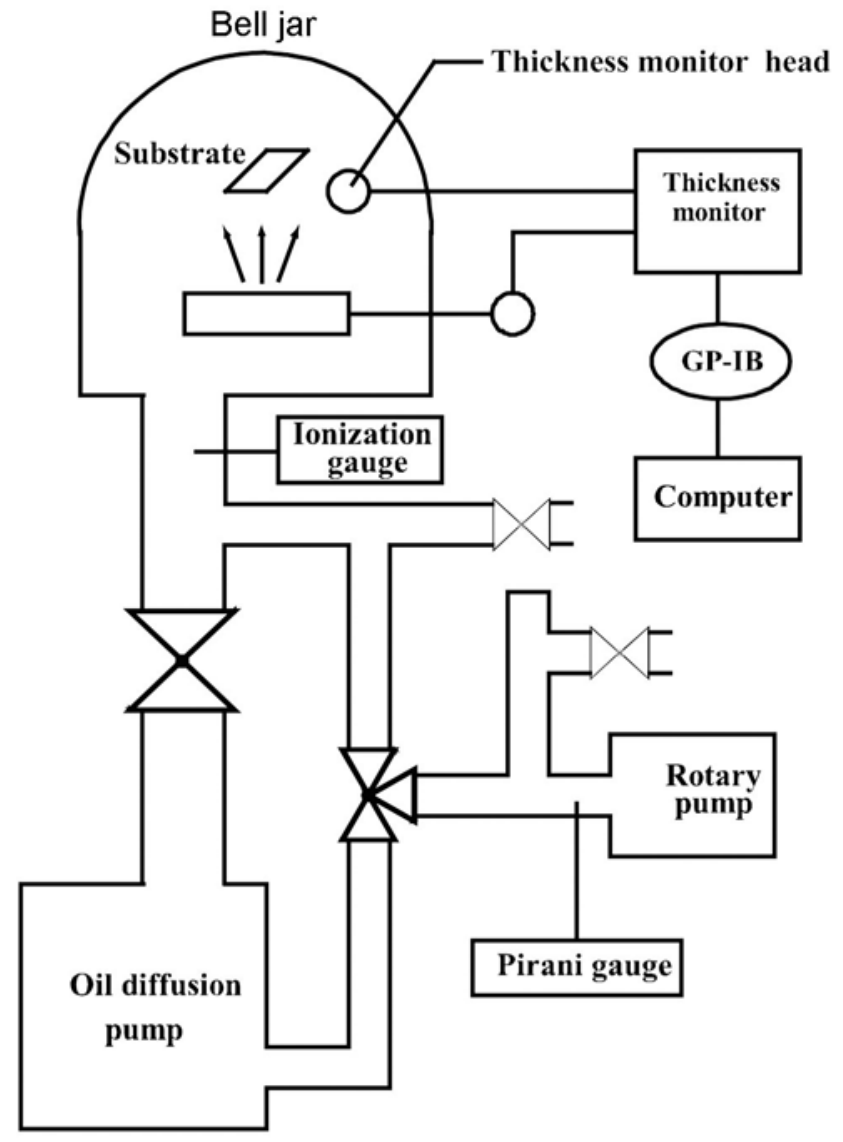

Figure 1. Schematic illustration of vacuum evaporation system.

order to remove the surface contaminations. The vacuum level of the chamber was attained at $3.0 \times 10^{-6}$ Torr before deposition and the glass substrates were kept at room temperature during the deposition. The deposition rate and target thickness of the deposited films were set to be $0.2 \mathrm{~nm} \mathrm{~s}^{-1}$ and $40 \mathrm{~nm}$, respectively. The morphology of the prepared $\mathrm{Au}$ substrates were checked by AFM. The film thickness and the dielectric constants of the Au layer were determined by ellipsometry.

\subsection{Experimental set-up for STM-LE measurement}

Figure 2 shows the experimental set-up employed to measure STM-LE in the prism-coupled geometry. Following the sample preparation process, the sample was pasted to the flat bottom of the hemispherical prism made of BK7 using an index-matching oil. The STM head shown in figure 2 was set over a rotation stage with the tip front over the rotation axis, which enabled us to detect STM-LE as a function of emission angle $\theta$ measured from the sample surface normal. The light emitted from the tip-sample gap in the direction of $\theta$ was collected by a lens with a focal length $f=70 \mathrm{~mm}$ and was focused 


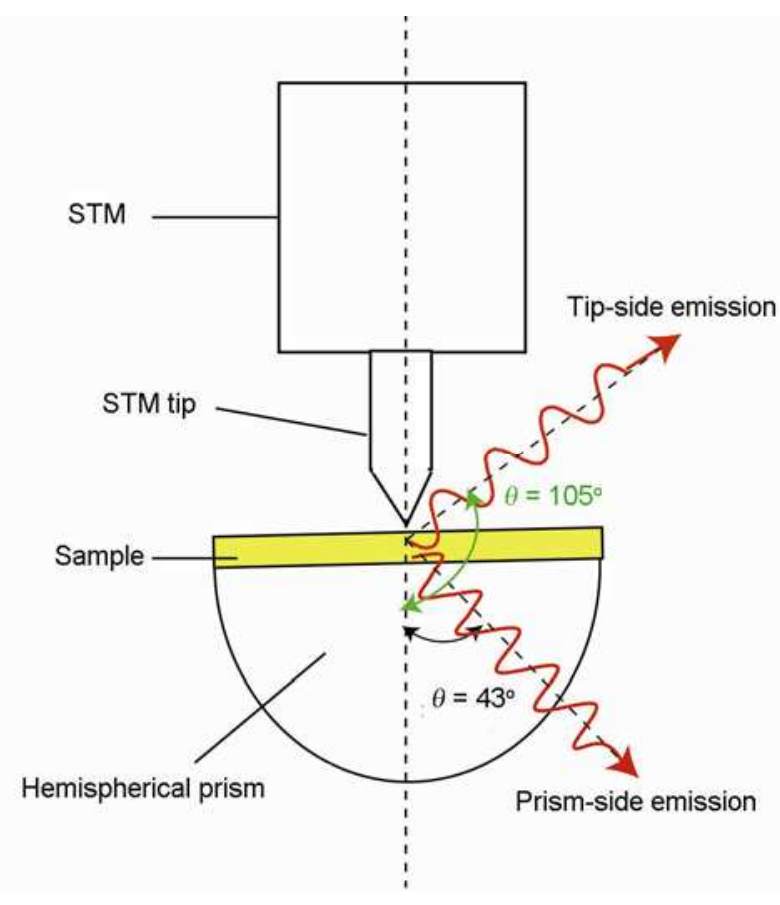

Figure 2. Schematic of experimental set-up for STM-LE measurements in the prism-coupled geometry.

by a lens with $f=150 \mathrm{~mm}$ onto the entrance surface of the optical fibre with a core diameter of $1 \mathrm{~nm}$. The light was then guided by the optical fibre to the spectroscopic system consisting of a spectrograph and a liquid-nitrogencooled charge-coupled device (CCD). The experimental spectra were taken for STM biasing conditions of $2.5 \mathrm{~V}$ and $2 \mathrm{nA}$ with an exposure time of $500 \mathrm{~s}$. The dark signals were subtracted from each experimental spectrum. All the experiments were carried out in air.

\section{Results and discussion}

Figure 3 shows the AFM images of 40-nm-thick gold films evaporated (a) at room temperature $\left(25^{\circ} \mathrm{C}\right)$ and on the preannealed $\left(450^{\circ} \mathrm{C}\right)$ glass substrates at the evaporation temperatures of (b) $25^{\circ} \mathrm{C}$, (c) $100^{\circ} \mathrm{C}$ and (d) $200^{\circ} \mathrm{C}$, respectively. It is clear from figure $3 \mathrm{a}$ and $\mathrm{b}$ that the relatively smooth surfaces were formed when the $\mathrm{Au}$ films were evaporated on the preannealed glass substrates. This indicates that the surface cleanliness obviously affects the growth process of the Au films. It is also observed from figure $3 \mathrm{~b}-\mathrm{d}$ that the flatness and also the discontinuity among the individual grains of the gold surfaces were increased when the glass substrates were kept at higher temperatures during the deposition of the Au films.

The morphology of the prepared Au substrate was checked by AFM. The film thickness and the dielectric constants of the $\mathrm{Au}$ layer were determined by ellipsometry. The typical surface-grain diameter as estimated from the

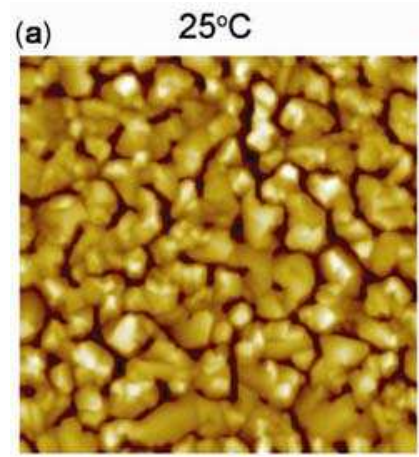

(b) $\quad 25^{\circ} \mathrm{C}$ (c)

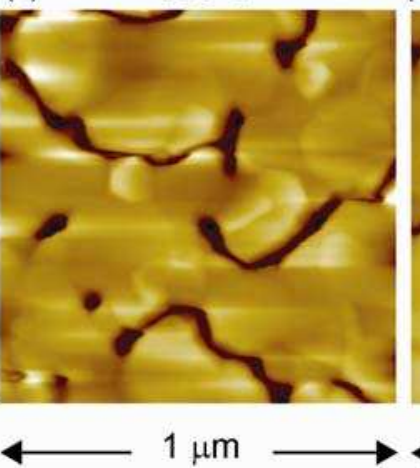

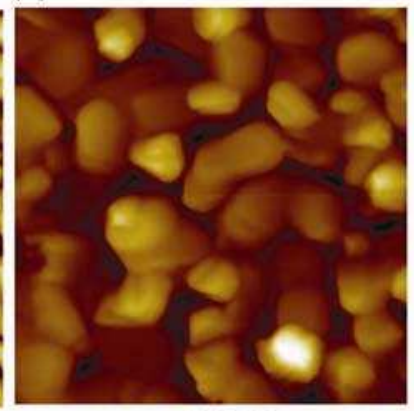

(d)



Figure 3. AFM images of (a) 40-nm-thick gold films evaporated at $25^{\circ} \mathrm{C}$ (room temperature) and (b) on the preannealed $\left(450^{\circ} \mathrm{C}\right)$ glass substrates at $25^{\circ} \mathrm{C},\left(\right.$ c) $100^{\circ} \mathrm{C}$ and (d) $200^{\circ} \mathrm{C}$.

AFM image of figure $3 \mathrm{~b}$ was found to $40 \mathrm{~nm}$ and the grain height was estimated to be around $5 \mathrm{~nm}$. Hence, the average characteristic of an evaporated $\mathrm{Au}$ film at room temperature has been obtained. Figure 4 shows the dielectric constants of the evaporated $\mathrm{Au}$ thin film as a function of photon energy; figure $4 \mathrm{a}$ shows the real part and figure $4 \mathrm{~b}$ shows the imaginary part of dielectric constant. The red line represents the experimental value and the blue line indicates the literature value. The work and dielectric functions were obtained from the literature. ${ }^{14-17}$ It is found from figure 4 that the real part of the dielectric constant of the evaporated gold film is almost agreed with the literature value. Although the result has been derived by using Kramers-Kroning relations from the real part of the dielectric functions of the gold film, there is a slight deviation in the imaginary part of the dielectric constant. Here, it is believed that the error was originated from the fact that the range of the measurement frequency was narrower in this experiment. Hence, it is concluded that the dielectric constant of the evaporated gold film showed the value as expected for the bulk gold film; moreover, the thickness of the gold film was estimated to be $40 \mathrm{~nm}$ by ellipsometry.

In order to clarify the experimental data, the STM-LE spectra in the prism-coupled geometry were calculated followed by a previously reported theoretical treatment. ${ }^{18}$ In this theoretical calculation, we have used the following 
equation, which is necessary to discuss the light emission mechanism.

$$
I=\frac{\omega^{6}}{64 \pi^{3} c^{5}} \cos ^{2} \theta_{0} \sum_{\mu=x, y, z} \varepsilon_{\mu z}^{*} \varepsilon_{\mu z} P_{z}^{*}(\omega) P_{z}(\omega),
$$

where $I$ is the radiation intensity, $c$ the speed of light, $P_{z}(\omega)$ the dipole, the dipole has only the surface normal component $z$ due to the geometrical symmetry. The summation is taken over the surface parallel components $x, y$ and the surface normal component $z$. $\theta_{0}$ is the observation angle measured from the surface normal, $\varepsilon_{\mu z}$ the electromagnetic Green's function matrix. The details of this calculations are described in ref. 18.

In this numerical calculation, the sample and tip materials were assumed to be $\mathrm{Au}$ and $\mathrm{W}$, respectively. The thickness of the Au film was set at $40 \mathrm{~nm}$ and the distance $d$ between the tip-front and the sample surface was assumed to be $1 \mathrm{~nm}$. The radius of the curvature of the tip-front $a$ was set at $100 \mathrm{~nm}$. The work and dielectric functions for these media were obtained from the literature. ${ }^{14-17}$ The refractive index of the prism is taken to



Figure 4. Dielectric constants of the evaporated $\mathrm{Au}$ thin film as a function of photon energy: (a) real part $\operatorname{Re}(\varepsilon)$ and (b) imaginary part $\operatorname{Im}(\varepsilon)$ of dielectric constant. be 1.51 for BK7 glass. The tunneling current and the bias voltage were fixed at $2 \mathrm{nA}$ and $2.5 \mathrm{~V}$, respectively. In this study, the parameter values were kept same both for the theoretical calculations and also for the experimental measurements. Figure 5 shows the theoretical calculated results of the light emission from the glass-gold interface. For this theoretical calculation, a three-layer structure which is consisted of vacuum-Au film with a 40-nmthick-BK7 prism with the light detection angle of $43^{\circ}$ were used. The dielectric function of BK7 was calculated at each frequency using the dispersion formula for this glass. The light emission intensity ratio as a function of photon energy is shown in figure 5a. From figure 5, it is clear that the intensity of the emitted photon is 10-100 times enhanced at the prism-coupled STM junction than that of a usual STM junction. The intensity of the emitted photon as a function of Au film thickness is also shown in figure $5 \mathrm{~b}$. From this theoretical calculated result (figure $5 b$ ), it is noticed that the light emission intensity is mostly enhanced when the Au film has a thickness of
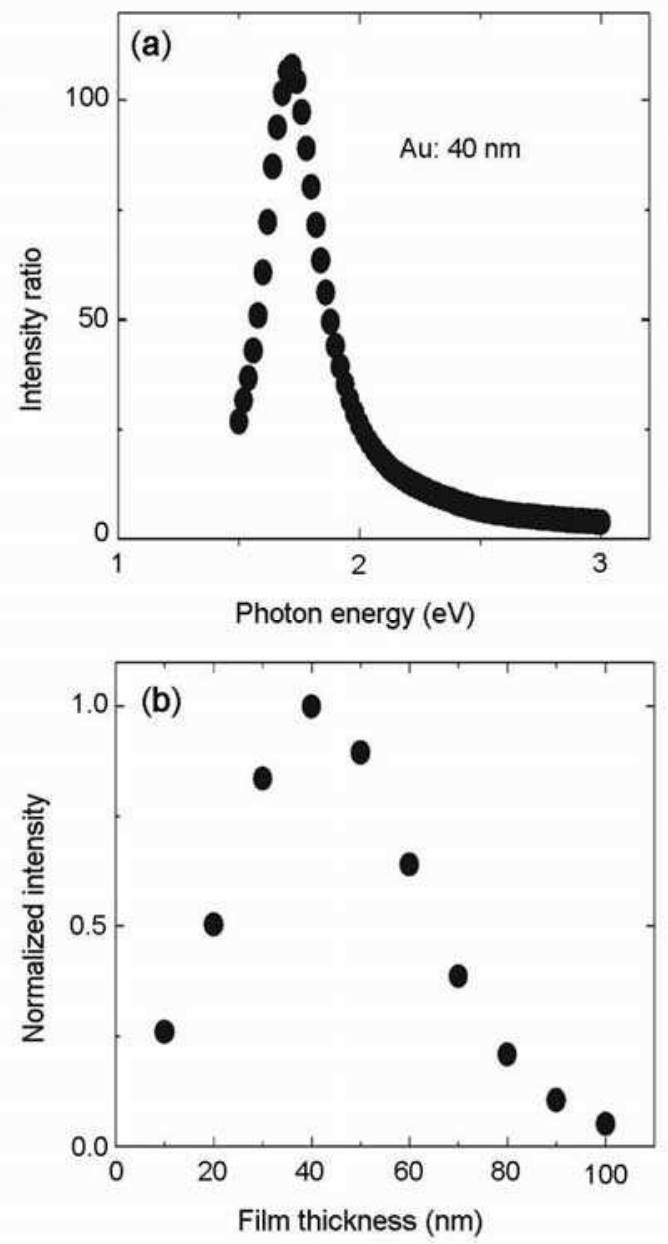

Figure 5. (a) The light emission intensity ratio as a function of photon energy. (b) The ratio was obtained by dividing the intensity of prism-coupled STM-LE by that of normal STM-LE and plots of the light emission intensity against the Au film thickness. 


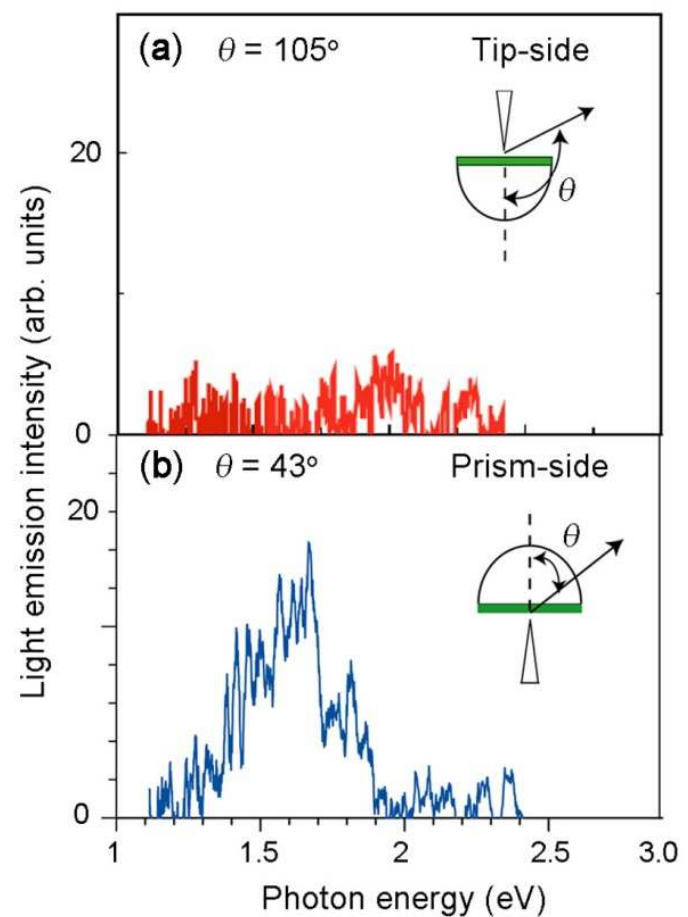

Figure 6. Experimental STM-LE spectra of the bare Au film. Results for emission angles of (a) $105^{\circ}$ (tip-side emission) and (b) $43^{\circ}$ (prism-side emission), respectively. The directions corresponding to these angles are depicted as arrows in the insets.

$40 \mathrm{~nm}$. Therefore, it is concluded that the $40-\mathrm{nm}$-thick gold film provides the most enhancement of light emission from the prism-coupled STM-LE.

After preparation and characterization of Au film, the film was deposited onto the flat bottom of a hemispherical prism, and the STM-LEs from the tip-sample gap into the vacuum (tip-side emission) and into the prism (prismside emission) were measured.

Figure $6 \mathrm{a}$ and $\mathrm{b}$ shows the experimental STM-LE spectra of the bare Au film for emission angles of $105^{\circ}$ (tip-side emission) and $43^{\circ}$ (prism-side emission), respectively. The directions corresponding to these angles are depicted as arrows in the insets. Despite the fact that both emissions are excited by the same electron tunneling, notable differences in total intensity (i.e., photon-energy integrated intensity) and spectral peak position are observed; the total intensity ratio of the tip-side emission (figure 6a) to the prism-side emission (figure 6b) is approximately $1: 8$, and their spectral peaks are positioned at 1.8 and $2.0 \mathrm{eV}$, respectively. It is known that the STMLE from metallic sample is radiated by LSPs excited by tunneling electrons. ${ }^{19}$ Although SPPs are simultaneously excited, ${ }^{13}$ they scarcely contribute to the signals in the conventional STM-LE measurements due to the existence of a wave vector mismatch between SPPs and light propagating in the vacuum. This mismatch prevents coupling between them on flat surfaces. ${ }^{20}$ In this study, we made
SPPs radiative by employing the prism-coupled (Kretschmann) geometry to eliminate the wave vector mismatch. ${ }^{21}$ As the light propagating in the glass has a wave vector whose magnitude is larger than that of SPPs localized at the metal-air interface, its wave vector component parallel to the interface can be matched to that of SPPs by adjusting the propagation angle of the light; hence, SPPs become radiative with the adjusted angle on the prism side. Hence, the spectral peak energies determined from figure $6 \mathrm{a}$ and $\mathrm{b}$ revealed that the tip-side emission is radiated by LSPs, and the prism-side emission is dominantly radiated by SPPs. Thus, the experimental finding that the prism-side emission is much stronger than the tip-side emission is indeed due to the geometry that makes SPPs radiative.

\section{Conclusions}

In order to investigate the STM-LE in the tip-sample gap region, gold films on glass substrates were evaporated in the vacuum evaporation system. AFM was used to probe the morphology of the evaporated Au films. AFM observation showed that the morphology of gold films strongly depends on the cleanliness of the glass substrates and the deposition temperature. Comparatively smooth surface was observed when a 40-nm-thick Au film was evaporated on a preannealed glass substrate at room temperature. Our theoretical calculation also showed that the light emission from the prism-coupled STM junction is strongly enhanced when the $\mathrm{Au}$ film has a thickness of $40 \mathrm{~nm}$. After preparation, the evaporated $\mathrm{Au}$ films were deposited on the flat bottom of a hemispherical glass prism; and the STM light emission from the tip-side and into the prism were measured. From the experimental result, it was found that the prism-side emission is much stronger than the tip-side emission because the prism-side emission is dominantly radiated by SPPs but not by LSPs.

\section{Acknowledgements}

Part of this work was carried out in the Nano-Photoelectronics Laboratory, Tohoku University, Japan, and was supported in part by the Tohoku University ElectroRelated Departments Global COE Program. We would like to thank Mr. Tomonori Sanbongi and Wataru Iida for their cooperation and technical assistance.

\section{References}

1. Pinsker Z G 1953 Electron diffraction (London: Butterworths Scientific Publications) p 258

2. Linnik V 1929 Nature 123604

3. Brück L 1936 Ann. Phys. Leipzig 26233

4. Rüdiger O 1937 Ann. Phys. Leipzig 30505

5. Buchholz S, Fuchs H and Rabe J P 1991 J. Vac. Sci. Technol. B 9857 
6. Coombs J H, Gimzewski J K, Reihl B, Sass J K and Schlittler R R 1998 J. Microsc. 152325

7. Uehara Y, Fujita T and Ushioda S 1999 Phys. Rev. Lett. 83 2445

8. Leite F L, Paterno L G, Borato C E, Herrmann P S P, Oliveira O N and Mattoso L H C 2005 Polymer 4612503

9. Mosiewicki M A, Schroeder W F, Leite F L, Herrmann P S P, Curvelo A A S, Aranguren M I and Borrajo J 2006 J. Mater. Sci. 4146154

10. Berndt R, Schlittler R R and Gimzewski J K 1991 J. Vac. Sci. Technol. B9 573

11. Katano S, Ushioda S and Uehara Y 2010 J. Phys. Chem. Lett. 12763

12. Uehara $\mathrm{Y}$, Iida T, Ito K, Iwami M and Ushioda S 2002 Phys. Rev. B 65155408
13. Takeuchi K, Uehara Y, Ushioda S and Morita S 1991 J. Vac. Sci. Technol. B 9557

14. Johnson P B and Christy R W 1972 Phys. Rev. B 64370

15. Lynch D W and Hunter W R 1985 Handbook of optical constants of solids (New York: Academic Press) p 357

16. Uda M, Nakamura A, Yamamoto T and Fujimoto Y 1998 J. Electron. Spectrosc. $\mathbf{8 8} 643$

17. Hopkins B J and Rivers J C 1963 Proc. Phys. Soc. 81590

18. Uehara Y, Kimura Y, Ushioda S and Takeuchi K 1992 Jpn. J. Appl. Phys. 312465

19. Berndt R, Gaisch R, Gimzewski K J, Reihl B, Schlittler R R, Schneider W D and Tschudy M 1993 Science 262 1425

20. Raether H 1988 Surface plasmons (Berlin: Springer) p 110

21. Kretschmann E 1971 Z. Phys. 241313 (in German) 